\title{
Transmission Characteristic Simulation of High Frequency Acousto-Optic Modulators
}

\author{
Suha Mousa Alawsi, Lubaba Abdul Kareem Al-Janabi, Soror A. Mahdi \\ Department of Physics, College of Sciences, Al-Nahrain University, Baghdad, Iraq \\ Email: suhaalawsi@gmail.com
}

How to cite this paper: Alawsi, S.M., Al-Janabi, L.A.K. and Mahdi, S.A. (2018) Transmission Characteristic Simulation of High Frequency Acousto-Optic Modulators. Optics and Photonics Journal, 8, 98-110. https://doi.org/10.4236/opj.2018.84010

Received: February 5, 2018

Accepted: April 27, 2018

Published: April 30, 2018

Copyright (C) 2018 by authors and Scientific Research Publishing Inc. This work is licensed under the Creative Commons Attribution International License (CC BY 4.0).

http://creativecommons.org/licenses/by/4.0/

\section{cc) (i) Open Access}

\begin{abstract}
The way by which one can make sure the operating mode of the modulation is by observing the Comsol results of the designed model of proposed acousto-optic modulator (AOM). These results include the pressure distribution, sound pressure distribution, stress distribution at piezoelectric, far-field analysis that describes the diffracted light orders, and electric potential versus light frequency. Throughout the simulating process of modulator operating using Comsol, it begins when the RF is power by a voltage of $100 \mathrm{~V}$, the light is then split into first ordered diffraction, which implies that the modulator is in the operating mode. The use of semiconductor materials is due to its smaller gap that easily transfers the energy that leads to generating first order diffraction when they provided a voltage power. It mentioned that zero order diffraction indicates the modulator does not run; other orders are appearing with increasing the frequency of light leading to decrease of the efficiency of the modulator performance.
\end{abstract}

\section{Keywords}

Acousto-Optic, Transmission of AO Modulator, High Frequency AOM, Crystal Material of AOM, Diffraction Efficiency of AOM

\section{Introduction}

The field of acousto-optics is describing the effect of light-sound interaction, which occurs because a sound wave induces localized refractive index variations in the medium through which it travels. This interaction is not just a curiosity but forms the basis of a whole range of practical devices. Moreover, the effect is relatively strong, and important parameters such as the amount of light reflected, the angle of deflection, and frequency shift are directly related to sound power and sound frequency, which can be controlled electronically. Such interaction by 
a mixing process that resulting add or difference frequency generation between phonons and photons [1]. These variations of acoustic wave parameters, including the amplitude, phase, frequency, and polarization are leading to modulate the optical wave. The acousto-optic interaction also makes it possible to modulate the optical beam by keeping the acoustic wavelength (frequency) fixed and varying the drive power to vary the amount of light in the deflected beam [2]. The acousto-optical devices are based on the effect of elesto-optic or photo-elastic (Raman and Nath, 1935; Klein, 1967). This interaction of AO happens in any optical medium when the optical beam and acoustic signal exist in that medium. When the AO crystal is injecting acoustic signal using piezoelectric mean, a strain will produce that changes the optical properties of the crystal. Then, the rarefraction and compression will generate a refractive index wave which is behaving like sinusoidal grating. Thus, the optical beam suffers some deflections, modulations, and frequency shifting when it passes through the crystal [3].

To understand fundamentals of $\mathrm{AO}$ interaction, a simple experiment is given to show the useful features required to exist in modern acousto-optic devices. The experimental setup is showing in Figure 1, it consists of a water-filled glass tank into which a sound wave frequency $f s$ is launch by a transducer [1]. The generated refractive index gives a diffraction grating moving with the velocity given by the speed of the sound wave in the medium. The light which then passes through the transparent material is diffracted due to this generated refraction index, forming a prominent diffraction pattern tilt at different angles from the original direction. The acousto-optic modulator (AOM) is a simple Bragg cell that uses the acousto-optic effect to diffract and shift the frequency of light using sound waves, which is used in lasers for Q-switching, telecommunications for signal modulation, and in spectroscopy for frequency control.

The far end of the tank is lined with a sound absorbing material to prevent reflections. When a collimated beam of light is made to impinge on the tank from the left, we observe that the light leaving the tank is split into many orders in the drawing. Upon increasing the sound power the number of orders visible is

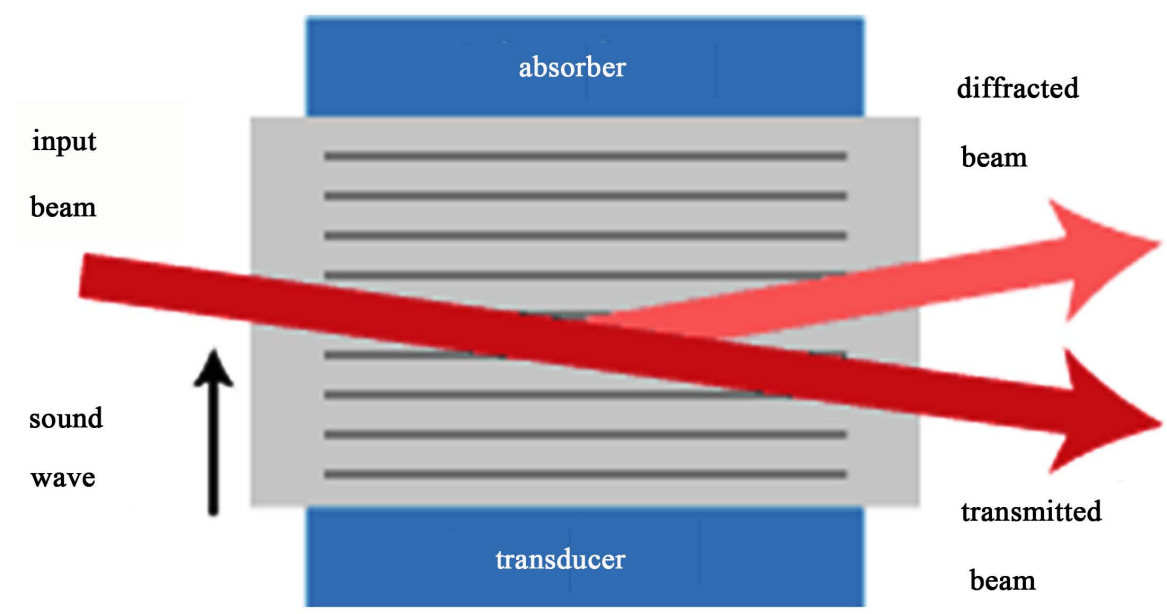

Figure 1. Experimental setup of AO. 
seen to increase gradually. The rarefactions and compressions in the sound wave induce a periodic change in the refractive index of the medium. The fact of moving the sound wave upward with sound velocity $\left(v_{s}\right)$ give a Doppler shift that determines the $n$th order is upshifted (or downshifted when $n$ is negative) by $n$ times the frequency of the sound. The simple experiment described above has revealed some very interesting phenomena which may well be put to use in a practical device: modulation, deflection, and frequency shifting. The state of art is essentially concerned with optimizing these effects for specific applications, which is achieved by analyzing the effect of certain parameters such as; the width of the sound beam (i.e., thickness of the induced grating), and the width of the incident beam of light relative to the wavelength of sound [1].

\section{Related Work and Contribution}

There are a lot of researchers related to the $\mathrm{AO}$ modulator design. The search for the right design is still continued to improve the modulations that used in the application fields. The following subsections illustrate the most interesting topics that introduce our contribution.

\subsection{Related Work}

Many works of literature are published in the field of $\mathrm{AO}$ modulator simulation and design. The differences between them are related to some aspects such as; modulator shape, used materials, power supply, wavelength, or even the application limitations. The two-dimensional theoretical model is proposed in [4] for strong acousto-optic interaction AOI of finite-sized beams with arbitrary profiles in acoustic field with curved wave front. The resulted numerical simulation of diffraction characteristics showed that the bandwidth and diffraction efficiency are increasing 1.5 times at least at weak interaction and more than five times at strong interaction during AOI (acousto-optic interaction) in the acoustic field of curved wave front [4]. In [5], this simulation of acousto-optic interaction is introduced and compared with experiment result by Mat lab program. Also, the accuracy of simulation was considered extensively to be used with a feedback circuit. Finally, a hybrid bitable optical device was designed to obtain the first-order and second-order feedback and getting physical insights of the system that observed in the simulation. While [6] derived and modified the equations of coupled-wave that describes the ultra-short optical pulses diffraction. There was a unified theory considering group delay between the Eigen waves in the crystal and anisotropic Bragg diffraction. The numerical results of the simulation showed an essential difference between of conventional theory and the modified one. It proves that precise characterization of acoustic-optical diffraction of femtosecond pulses needs solving the problem of Bragg coupling in bi-refringent media of wave packets. Also, [7] proposed a fast and right method for calculating AO figure of Merit (AOFM). This method permits to calculate the distribution of AOFM spatially and also the acoustic frequency for both un- 
iaxial and biaxial crystals. The obtained results of using the crystals $\mathrm{TeO}_{2}, \mathrm{LiNbO}_{3}$, $\mathrm{Te}, \mathrm{SrB}_{4} \mathrm{O}_{7}$, and $\mathrm{PbB}_{4} \mathrm{O}_{7}$ are identified that mentioned in works of literature, and ensure the validity and applicability of the proposed method [7]. To explore the interaction between the ultrasound and light as that found in $\mathrm{AO}$. The interaction between ultrasonic and light point source in a medium is exhibiting meta-acoustic properties that heuristically analyzed by assuming perfect imaging of the point source in the meta acoustic medium. The theoretical concepts of Raman-Nath and Bragg diffractions besides the interaction between non-propagating ultrasound and light are developed [8].

\subsection{Contribution}

The present work concerned with determining the requirements by which can design an acousto-optic modulator. This enables to estimate the transmission characteristics of the designed modulator for simulating its performance, and then the simulation leads to improve the performance efficiency. Also, the simulation enables to work the designed $\mathrm{AO}$ in different situations with these variations some determined parameters. The use of four different types of materials enables to measure the acousto-optic properties that may affect the performance efficiency of the proposed $\mathrm{AO}$ design.

\section{Proposed AOM Design}

The proposed AOM design is implementing using Comsol software. Comsol provides some pre-established tools for designing and simulating the performance of the acousto-optic modulators, in which one can set the boundary conditions that make the operating situation of the designed AOM more realize. The proposed AOM design is shown in Figure 2, there is an acousto-optic medium of dimensions $3 \times 4 \mathrm{~cm}$ and $3 \times 0.5 \mathrm{~cm}$, the dimension of the piezoelectric transducer is chosen from PZT-5H material (Lead Zirconate Titanate) that is commonly used the material in piezoelectric transducers. Whereas, the optic crystal domain is considered in three cases according to the used material: the first is the use of Lithium Niobate (LiNbO3), the second is the use of fused silica $\left(\mathrm{SiO}_{3}\right)$, while the third is the use of Germanium $(\mathrm{Ge})$, and the fourth one is the use of Tellurium dioxide $\left(\mathrm{TeO}_{2}\right)$. In this model, a single crystal plate is simulated with keeping the rotational symmetry of the element.

The material of the piezoelectric is model solving the Solid Mechanics and Electrostatics physics that are coupled via the linear constitutive equations that relating stresses and strains to electric displacement and electric field. These physics interfaces solve for the balance of body forces and volume charge density respectively as Equation (1). This coupling is automatically implemented in COMSOL Multi-physics by the Piezoelectric Effect node located under the Multi-physics branch in the Model Builder. The settings of the boundary conditions for the modulator model that initially build are leads to determine the characteristics of the designed modulator. 


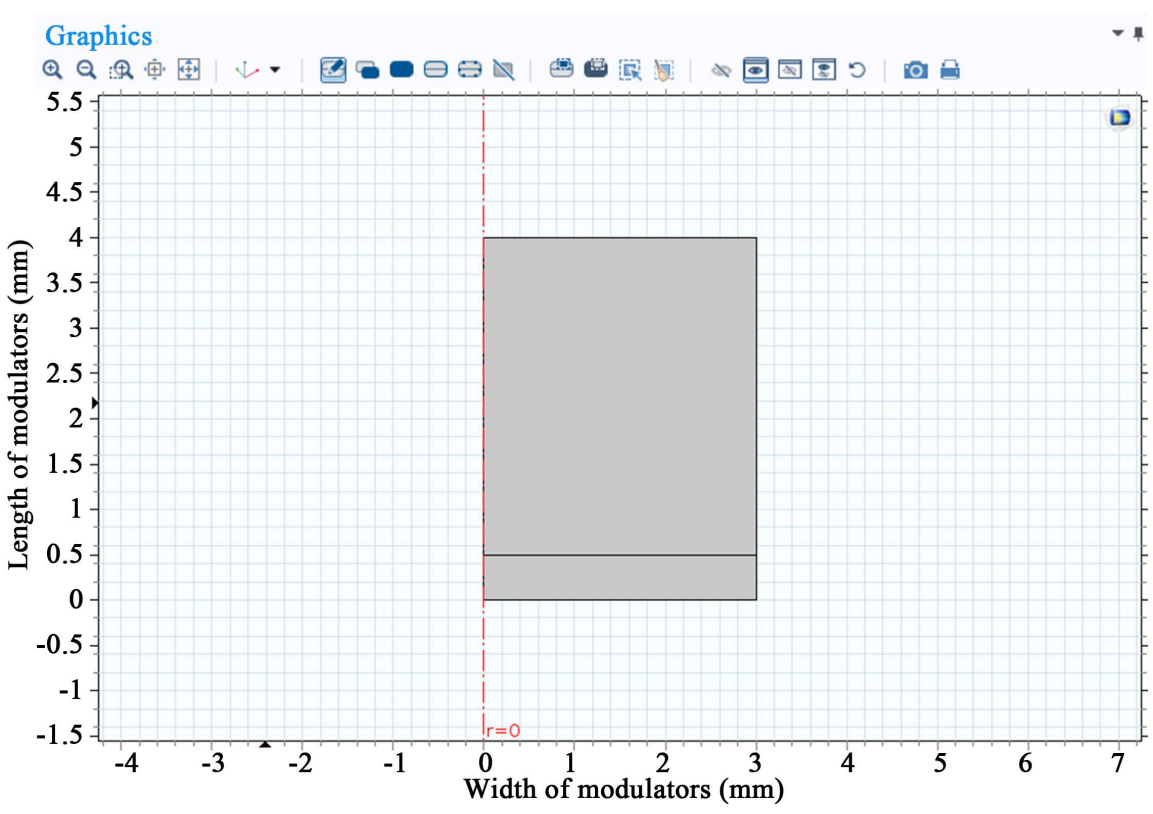

Figure 2. Geometry of proposed acousto-optic modulator design.

$$
\nabla \cdot D=0 \text { and } \nabla \cdot \sigma=0
$$

Table 1 presents the operating parameters of the proposed AO modulator. These parameters are input to Comsol at the model building stage. These parameters are optimized by trial and error to achieve the optimal value of each measure given in the table. The first three parameters are related to the modulator size, then the advice power is determined as the fourth parameter, the fifth parameter determines the place of interaction in the designed modulator, then the wavelength of the used light is determined as the sixth measure, the seventh and eighth parameters are related to the room temperature that determines the temperature of the modulator environment. Due to it is intended to use different crystal material, there are five operating parameters are determined of each material, the first is the material density, the second is the refractive index of the material, the third is the acoustic velocity that measured according to Equation (2) Where $d$ represents the diameters of the optical beam waist. Higher modulator speed is obtained when $t$ is as small as possible, that indicates the modulation speed, the fourth one is the figure of Merit, while the fifth is the attenuation. Except for the acoustic velocity, the remaining four parameters are constants for each material, while the acoustic velocity is varying according to the rise time and beam diameter.

$$
v_{a}=a / t
$$

\section{Results and Discussions}

Figure 3 shows the acoustic pressure distribution for the Lithium Niobate $\left(\mathrm{LiNbO}_{3}\right)$ material domain at different frequencies, in which the piezoelectric transducers type is Lead Zirconate Titanate (PZT-5H). The colored scale on the 
Table 1. Operating parameters of proposed AOM for different materials.

\begin{tabular}{|c|c|c|}
\hline \multicolumn{2}{|c|}{ Operating parameter } & Value and unit \\
\hline \multicolumn{2}{|c|}{ Interaction length $(\mathrm{L})$} & $\mathrm{L}=3 \mathrm{~cm}$ \\
\hline \multicolumn{2}{|c|}{ Modulator height $(\mathrm{H})$} & $\mathrm{H}=4 \mathrm{~cm}$ \\
\hline \multicolumn{2}{|c|}{ Laser beam diameter (D) } & $\mathrm{D}=1 \mathrm{~mm}$ \\
\hline \multicolumn{2}{|c|}{ Acoustic power $\left(\mathrm{P}_{\mathrm{a}}\right)$} & 0.5 watt $\leq \mathrm{P}_{\mathrm{a}} \leq 2.5$ watt \\
\hline \multicolumn{2}{|c|}{ Focal length $(F)$} & $\mathrm{F}=3 \mathrm{~mm}$ \\
\hline \multicolumn{2}{|c|}{ Operating signal laser wave length $(\lambda)$} & $\lambda=1.6 \mu \mathrm{m}$ \\
\hline \multicolumn{2}{|c|}{ Room temperature $\left(\mathrm{T}_{0}\right)$} & $\mathrm{T}_{0}=300 \mathrm{~K}$ \\
\hline \multicolumn{2}{|c|}{ Ambient temperature $(\mathrm{T})$} & $300 \mathrm{~K} \leq \mathrm{T} \leq 340 \mathrm{~K}$ \\
\hline \multirow{6}{*}{$\begin{array}{c}\mathrm{LiNbO}_{3} \\
\text { Lithium niobate } \\
\text { Based AOM device }\end{array}$} & Material density & $\rho=4644$ \\
\hline & Refractive index & $\mathrm{n}=2.2$ \\
\hline & Acoustic velocity & $\mathrm{v}_{\mathrm{a}}=6.6 * 10^{3} \mathrm{~m} / \mathrm{sec}$ \\
\hline & Figure of merit & $\mathrm{M}_{2}=7 \mathrm{~cm}^{2} / \mathrm{sec}$ \\
\hline & Attenuation & $\alpha=0.15 \mathrm{~dB} / \mathrm{GH}_{\mathrm{Z} . \mathrm{cm}}$ \\
\hline & Material density & $\rho=5606$ \\
\hline \multirow{4}{*}{$\begin{array}{c}\mathrm{SiO}_{2} \\
\text { Fused silica } \\
\text { Based AOM device }\end{array}$} & Refractive index & $\mathrm{n}=1.46$ \\
\hline & Acoustic velocity & $\mathrm{v}_{\mathrm{a}}=5.96 * 10^{3} \mathrm{~m} / \mathrm{sec}$ \\
\hline & Figure of merit & $\mathrm{M}_{2}=1.5 \mathrm{~cm}^{2} / \mathrm{sec}$ \\
\hline & Attenuation & $\alpha=6.45 \mathrm{~dB} / \mathrm{GH}_{\mathrm{Z} . \mathrm{cm}}$ \\
\hline \multirow{6}{*}{$\begin{array}{c}\mathrm{TeO}_{2} \\
\text { Tellurium dioxide } \\
\text { Based AOM device }\end{array}$} & Material density & $\rho=4260$ \\
\hline & Refractive index & $\mathrm{n}=2.25$ \\
\hline & Acoustic velocity & $\mathrm{v}_{\mathrm{a}}=4.26^{*} 10^{3}$ \\
\hline & Figure of merit & $\mathrm{M}_{2}=34$ \\
\hline & Attenuation & $\alpha=4.75 \mathrm{~dB} / \mathrm{GH}_{\mathrm{Z} . \mathrm{cm}}$ \\
\hline & Material density & $\rho=5323 \mathrm{~kg} / \mathrm{m}^{3}$ \\
\hline \multirow{4}{*}{$\begin{array}{c}\mathrm{Ge} \\
\text { Germanium } \\
\text { Based AOM device }\end{array}$} & Refractive index & $\mathrm{n}=4$ \\
\hline & Acoustic velocity & $\mathrm{v}_{\mathrm{a}}=5.5^{*} 10^{3}$ \\
\hline & Figure of merit & $\mathrm{M}_{2}=180$ \\
\hline & Attenuation & $\alpha=10.5 \mathrm{~dB} / \mathrm{GH}_{\mathrm{Z} . \mathrm{cm}}$ \\
\hline
\end{tabular}

side of each distribution represents the acoustic pressure values measured in Pascal $(P a)$ that plotted along the $\mathrm{z}$-axis, while the horizontal plane is presenting the modulator dimensions. It is shown that the acoustic pressure varies with the variation of frequency, where the largest values of acoustic pressure are: 8.95, $6.49,16.5,20.4 \times 10^{6} \mathrm{~Pa}$ for the four adopted frequency values $200,300,400$, and $500 \mathrm{kHz}$ as shown in Figure 4.

It is shown that the behavior of acoustic pressure is proportional to the frequency, and its maximum value is $20.4 \times 10^{6} \mathrm{~Pa}$ found at $500 \mathrm{kHz}$, in which the 


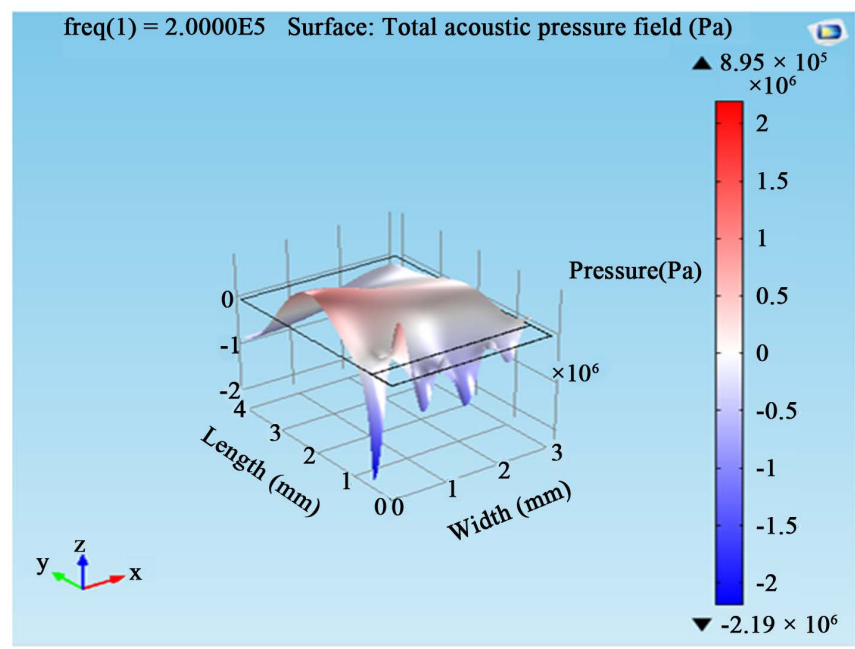

(a)

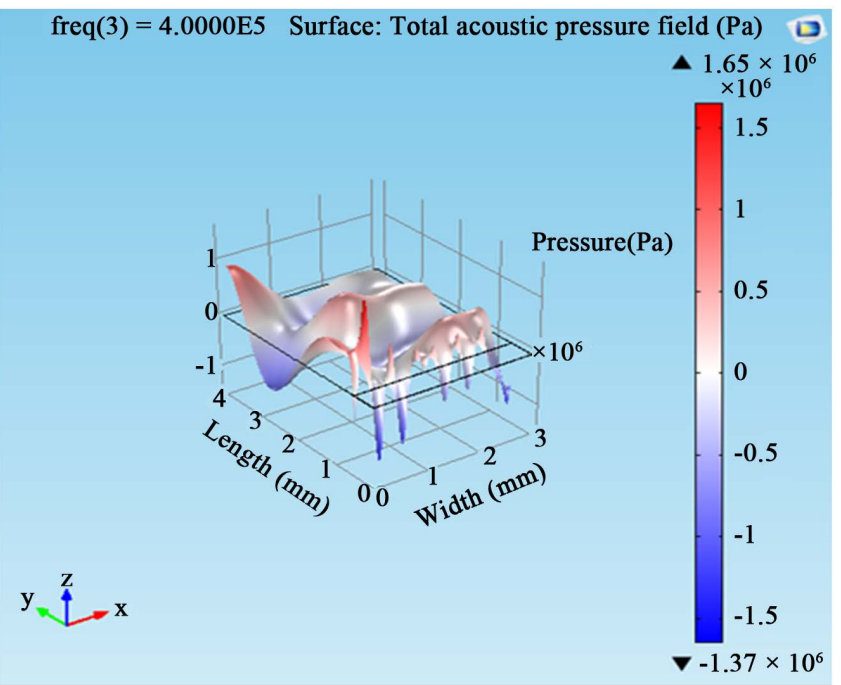

(c)

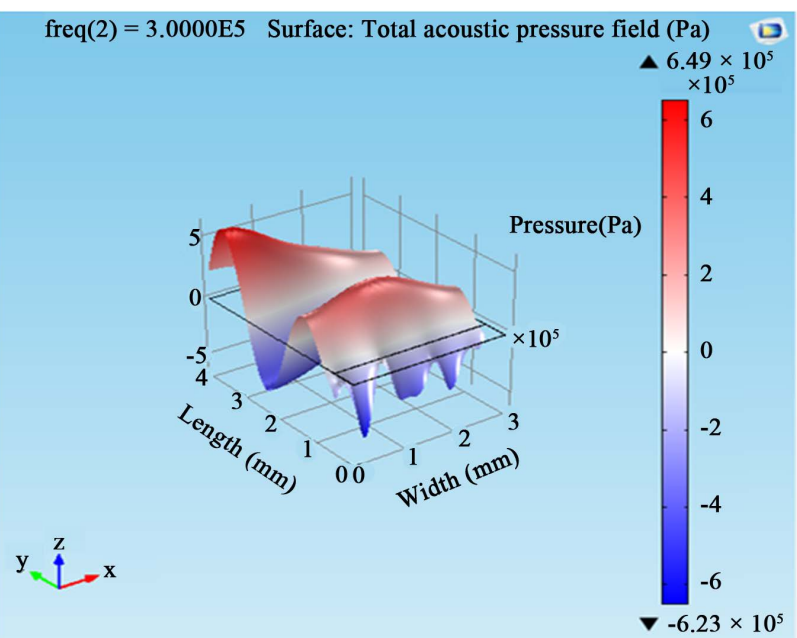

(b)

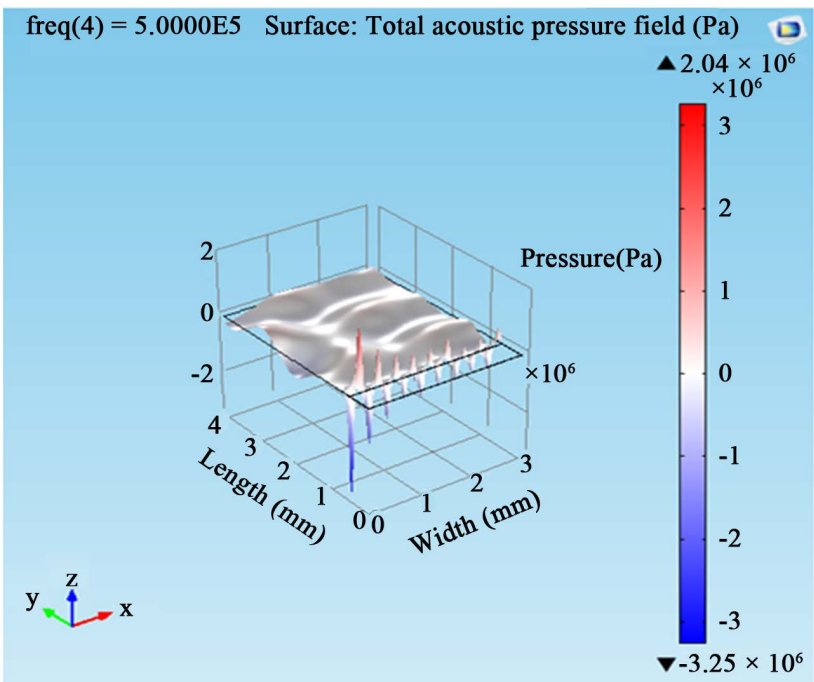

(d)

Figure 3. The acoustic pressure distribution for the lithium niobate.

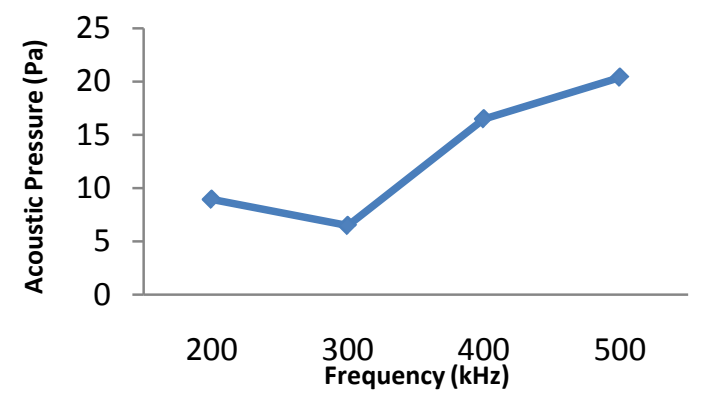

Figure 4. Maximum acoustic pressure values versus frequency for lithium niobate.

efficiency is highest due to the increase in the frequency leads to increase the power of acoustic that directly proportional to the efficiency. Figure 5 shows the sound pressure distribution in the acoustic crystal domain for the same different 


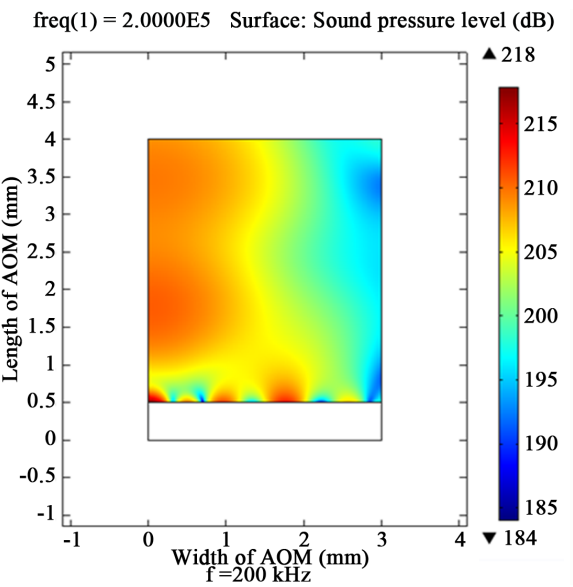

(a)

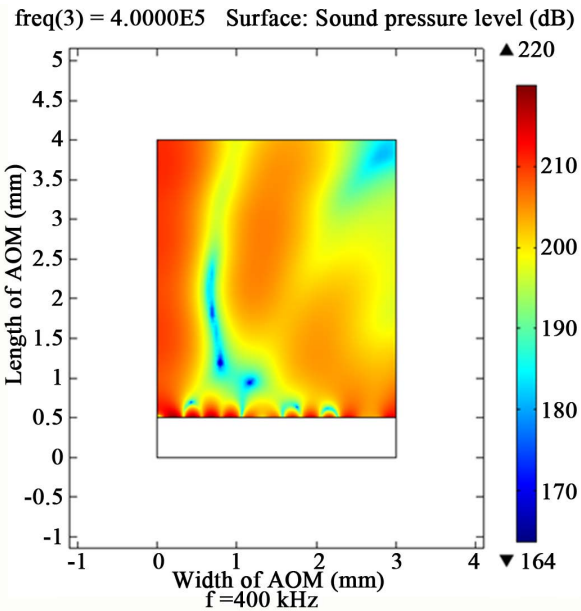

(c)

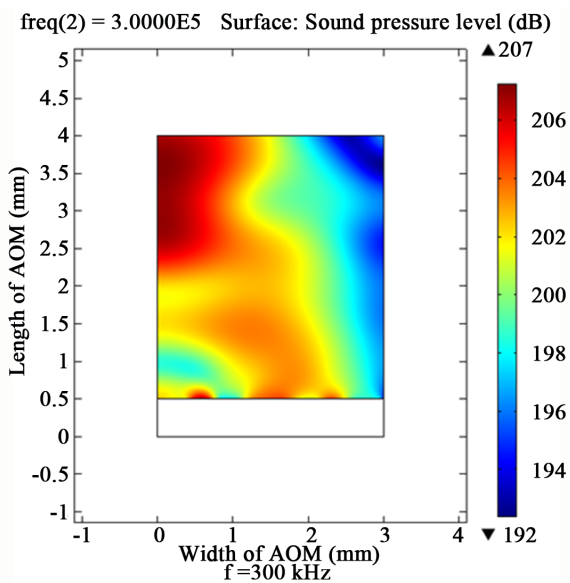

(b)

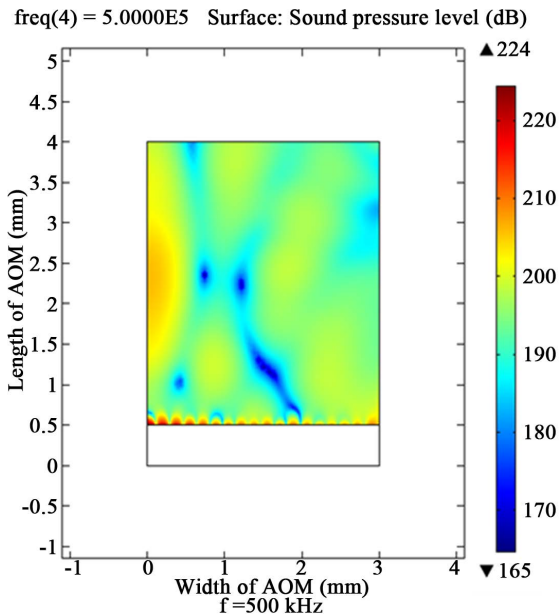

(d)

Figure 5. Sound pressure distribution in the acoustic crystal domain.

values of frequencies. The color scale in the side of figure represents the sound pressure levels distributed along the modulator dimension.

It is shown that the sound pressure varies with the variation of frequency, where the largest values of sound pressure are: 218, 207, 220, 224 decibel ( $d B)$ for the four adopted frequency values $200,300,400$, and $500 \mathrm{kHz}$ successively as shown in Figure 6. It is noticeable that the behavior of sound pressure is directly proportional to the frequency and its largest value is $224 \mathrm{~dB}$ found at $500 \mathrm{kHz}$, where the efficiency is also highest for the same reason of acoustic pressure variation mentioned before. It should be mentioned that the distribution of the acoustic pressure is transverse along the modulator dimension, while the sound pressure is distributed longitudinal. The decrease occurs in the sound pressure at $300 \mathrm{kHz}$ on its value at other frequencies is due to its perpendicularly to the acoustic pressure.

Furthermore, Figure 7 shows the stress distribution in the piezoelectric domain for the same considered values of frequency. This distribution is extended only along the transducers dimensions at the bottom of modulator crystal. The 


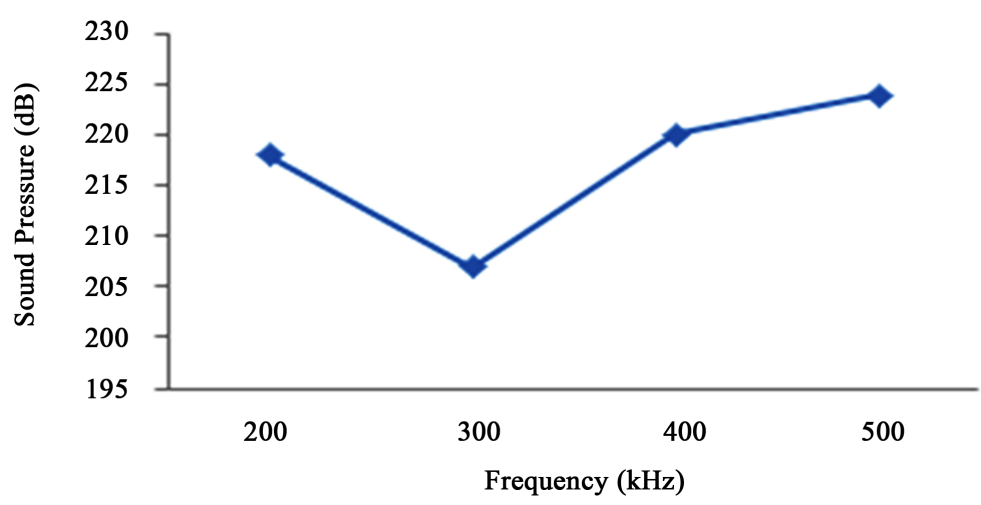

Figure 6. Maximum sound pressure values versus frequency for lithium niobate.

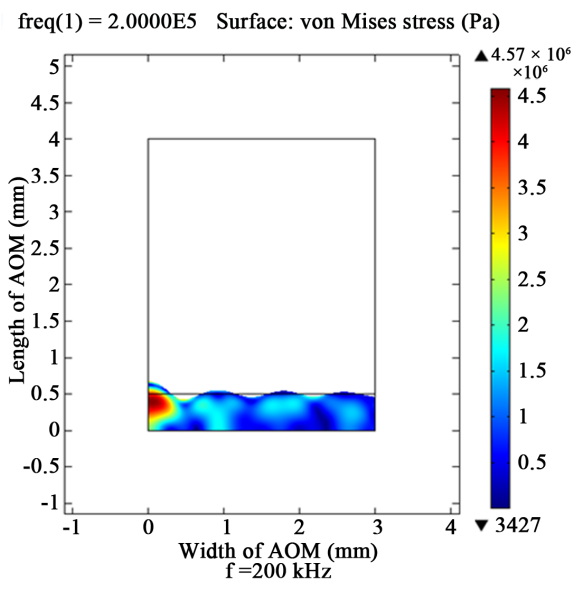

(a)

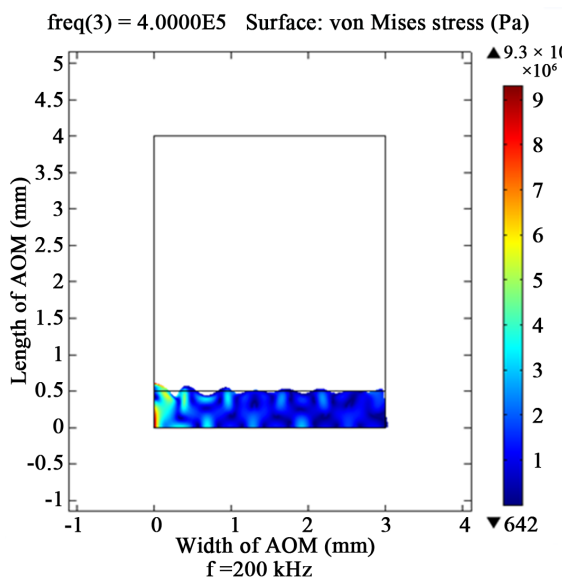

(c)

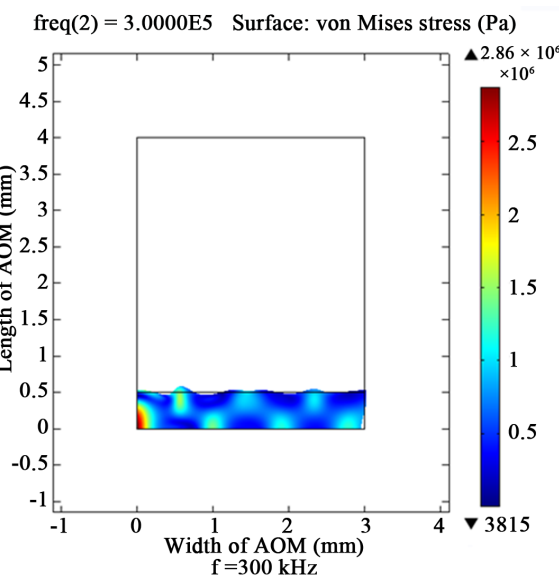

(b)

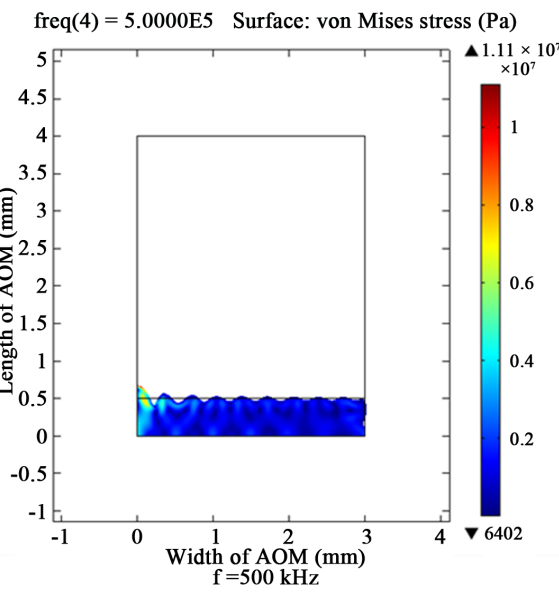

(d)

Figure 7. Stress distribution in the piezoelectric domain.

color scale at the side of each distribution graph refers to the amount of stress that extended along piezoelectric area (i.e. $3 \mathrm{~mm}$ width and $0.5 \mathrm{~mm}$ height), the vertical axis of the graph refers to the height of transducers, while the horizontal axis refers to the width of the transducers. It is noticeable that the stress varies 
with the variation of frequency, where the largest values of the stress are: 4.57, $2.86,9.3$, and $111.0 \times 10^{6} \mathrm{~Pa}$ for the four considered frequency values 200,300 , 400 , and $500 \mathrm{kHz}$ as shown in Figure 8. It is shown that the behavior of stress is proportional to the frequency, and its largest value is $111.0 \times 10^{6} \mathrm{~Pa}$ found at 500 $\mathrm{kHz}$, where the efficiency is the highest value. The decrease occurs in the stress at $300 \mathrm{kHz}$ on its value at other frequencies is due to its relations with the sound and acoustic pressures.

Figure 9 shows the one-dimensional behavior of stress distribution the four considered frequencies: 200,300, 400, and $500 \mathrm{kHz}$, where the vertical axis represents the stress values while the horizontal axis represents the width of the modulator. It is noticeable that the largest stress value is found the region at $r=$ $0-0.2 \mathrm{~mm}$, this distance is a part of piezoelectric width extended along the length of transducers. Then, the stress value decreases the region of a width greater than $0.2 \mathrm{~mm}$ till reaching the end of transducers at $3 \mathrm{~mm}$. This is due to such region is the effective one that makes the RF to operated. The two bold points appeared in Figure 9 refers to the horizontal shifting that indicates the distance between the zero order and first order diffraction, which can be noticed only at the stage of RF operating. The estimated shift is about $1 \mathrm{~mm}$ with very little changes may occur with the change of the frequency. As increasing the number of orders, the efficiency of the modulator performance will be increased. The angles between the shifted light beams are relatively little due to plenty of diffracted beams are occurred.

Figure 10 shows the variations of electric potential, the color scale at the side of.

Figure 9 shows the behavior of stress distribution the four considered frequencies.

The graph refers to the electric potential values, while the vertical and horizontal dimensions point to the width and height of the modulator. The four graphs show the electric potential behavior for the four considered frequencies.

The electric potential is directly provided to the RF circuit and then distributed on the piezoelectric. It is noticeable that the electric potential at $r=0.2$

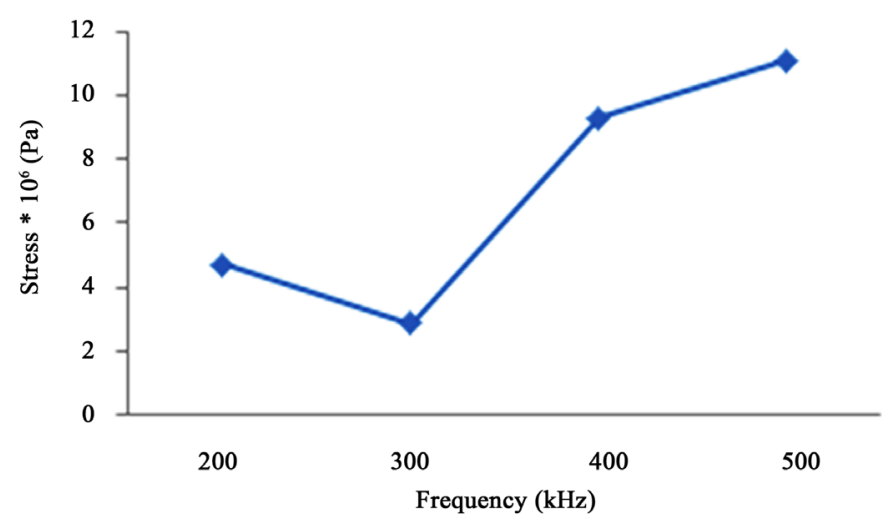

Figure 8. Maximum stress values versus frequency for lithium niobate. 


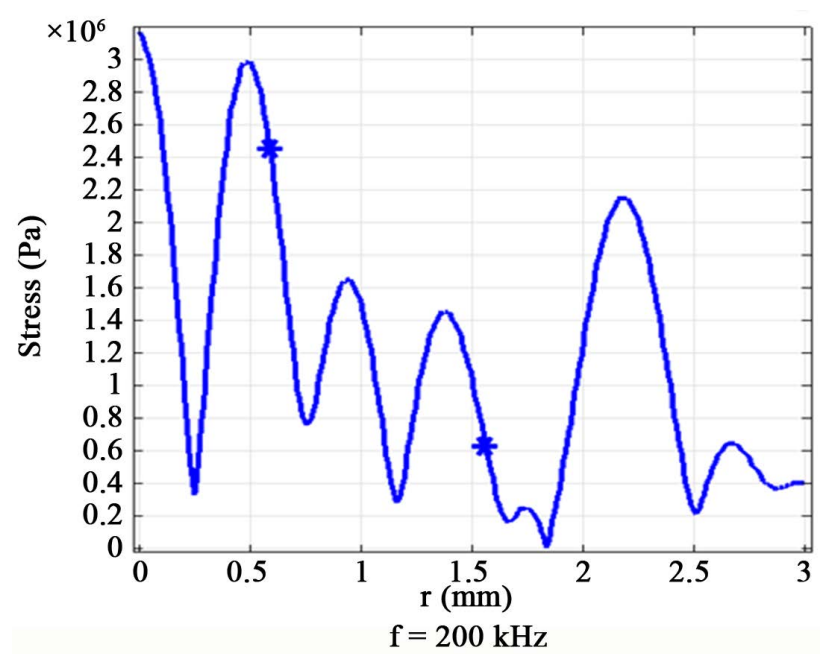

(a)

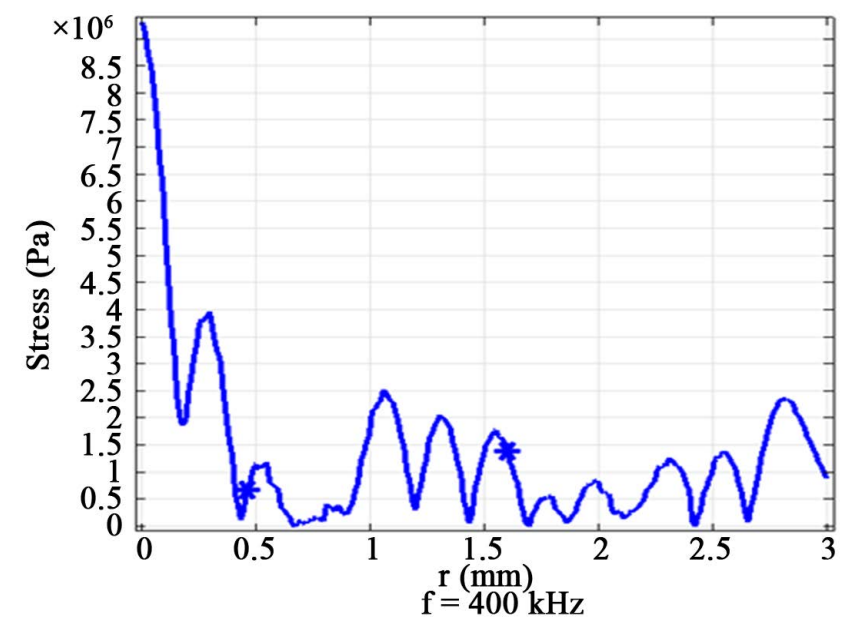

(c)

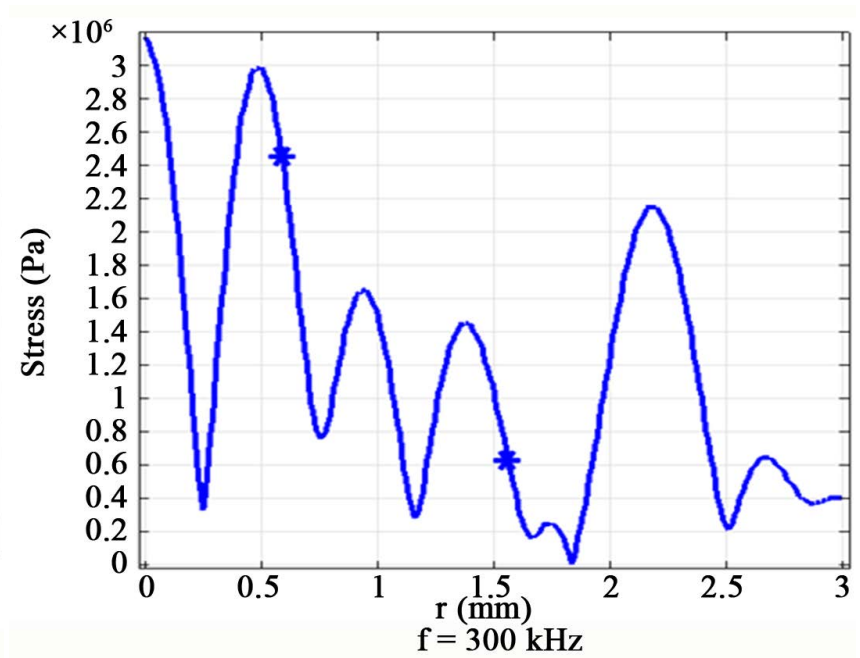

(b)

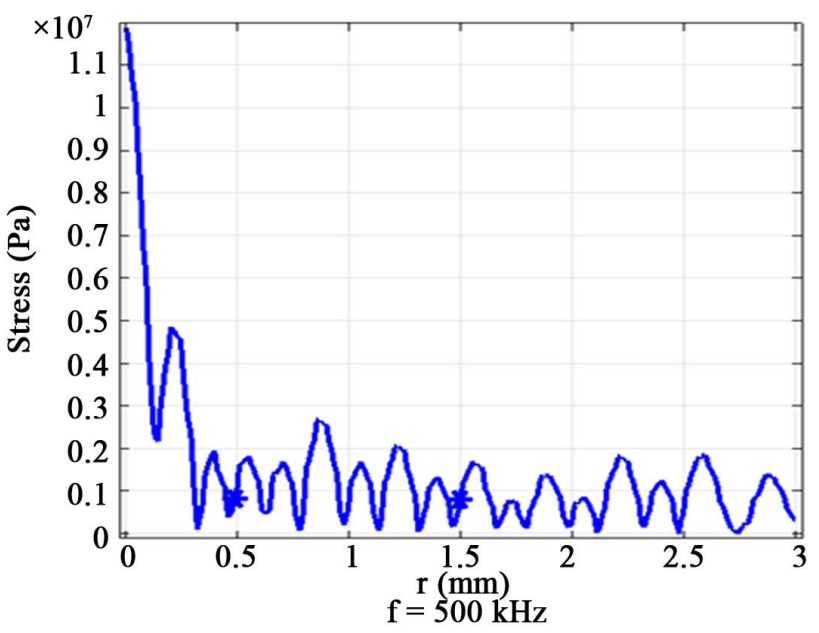

(d)

Figure 9. Stress distribution the four considered frequencies.

$\mathrm{mm}$ is increasing from zero up to $150 \mathrm{~V}$ at a $200 \mathrm{kHz}$ frequency, it is increasing from zero up to $106 \mathrm{~V}$, it is increasing from zero up to $164 \mathrm{~V}$ and it is increasing from zero up to $177 \mathrm{~V}$, as Figure 11 shows.

\section{Conclusions}

The most important conclusions that indicated throughout the implementation of the present work are given in the following:

1) The acoustic distribution and sound distribution in acoustic crystal give good diffraction efficiency at the $500 \mathrm{kHz}$ frequency.

2) The stress distribution in piezoelectric transducers gives good efficiency at the $500 \mathrm{kHz}$ frequency, the increase of light frequency leads to increase of the modulator performance efficiency.

3) The largest stress value is found at $0.2 \mathrm{~mm}$ width of piezoelectricity, which is decreasing gradually till reaching $3 \mathrm{~mm}$ width. 


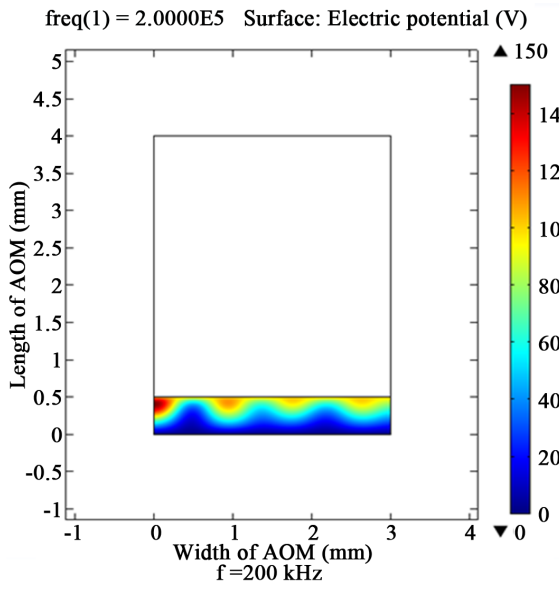

(a)

freq $(3)=4.0000 \mathrm{E} 5$ Surface: Electric potential $(\mathrm{V})$

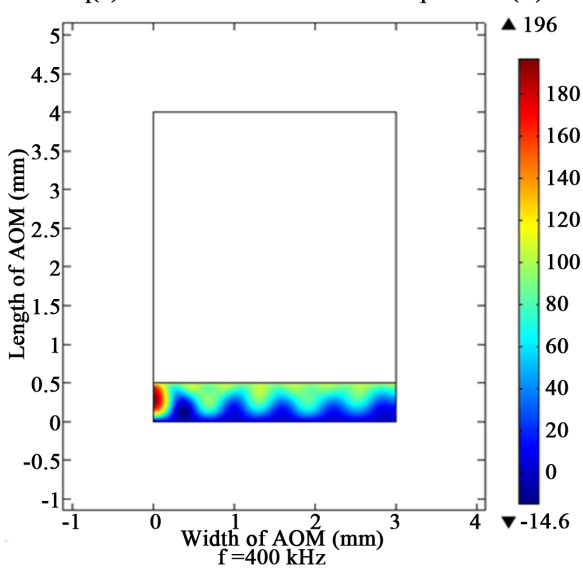

(c) freq $(2)=3.0000 \mathrm{E} 5$ Surface: Electric potential $(\mathrm{V})$

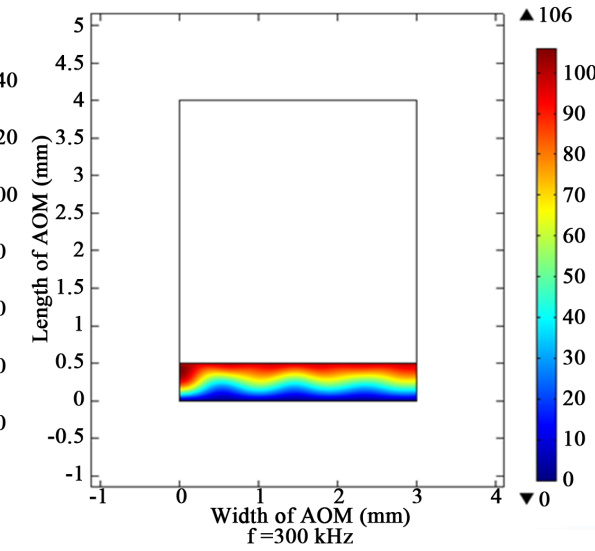

(b)

freq $(4)=5.0000 \mathrm{E} 5$ Surface: Electric potential $(\mathrm{V})$

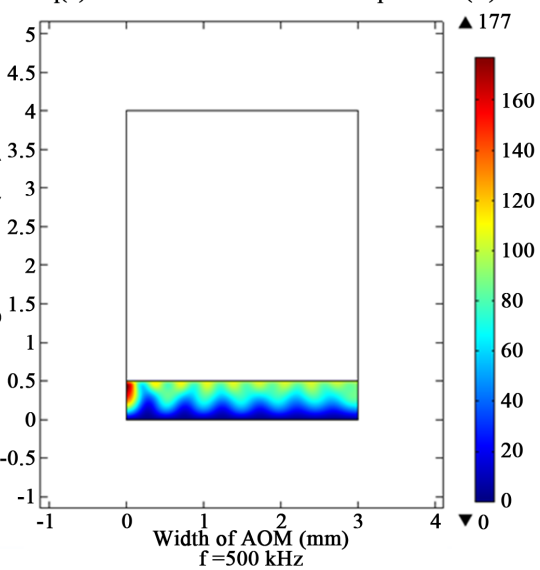

(d)

Figure 10. Variation of electric potential with the frequency variation.

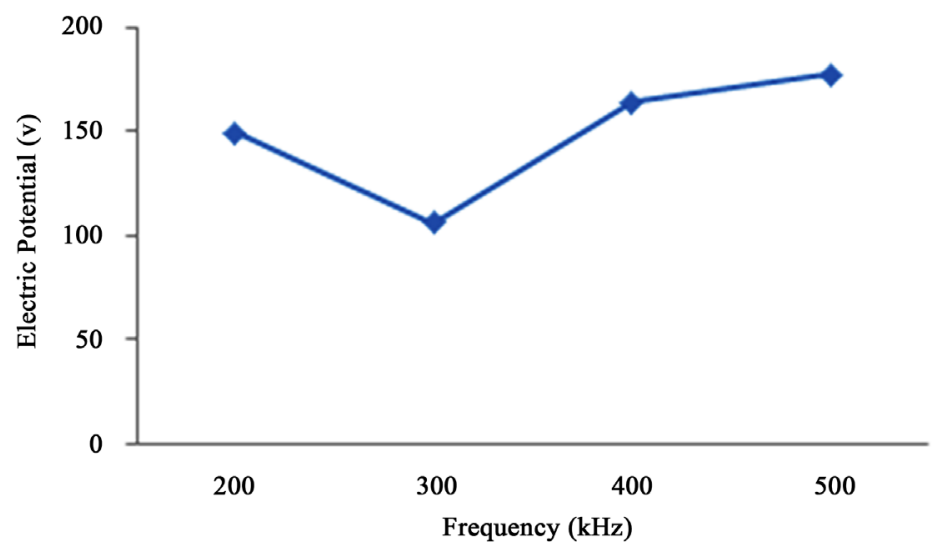

Figure 11. Electric potential variation with frequencies.

4) At the width of $0.2 \mathrm{~mm}$ of transducers, the horizontal shift has appeared with the little effect to the frequency.

5) The largest efficiency based on far-field analysis occurs in $500 \mathrm{kHz}$ frequency. 
6) In spite of more orders appeared in the diffracted light, the diffracted light is shown with greater diffracted angles, which leads to decrease of the efficiency.

7) The electric potential reaches its largest value at $500 \mathrm{kHz}$.

8) The diffraction efficiency increases with the acoustic power.

\section{References}

[1] Saleh, B.E.A. and Teich, M.C. (2013) Fundamentals of Photonics. 2nd Edition, John Wiley \& Sons, Hoboken.

[2] Mayden, D. (2002) Acousto Optical Pulse Modulators. Journal of Quantum Electronics, 6, 15-24. https://doi.org/10.1109/JQE.1970.1076309

[3] Silva, R., Hartung, A., Rothhardt, M., Pohl, A.A. and Bartelt, H. (2013) Simulation of Acousto-Optical Modulation of Fiber Bragg Gratings in Suspended Core Fibers. Workshop on Specialty Optical Fibers and Their Applications, Paper F2.22. https://doi.org/10.1364/WSOF.2013.F2.22

[4] Chang, I. (1976) Acousto-Optic Devices and Application. IEEE Transactions on Sonics and Ultrasonic, 23, 2. https://doi.org/10.1109/T-SU.1976.30835

[5] Young, E.H. and Yao, S.-K. (1981) Design Considerations for Acousto-Optic Devices. Proceedings of the IEEE, 69, 54-64. https://doi.org/10.1109/PROC.1981.11920

[6] Gazalet, M., Kastelik, J., Bruneel, C., Bazzi, O. and Bridoux, E. (1993) Acousto-Optic Multi-Frequency Modulators Reduction of the Phase-Grating Intermodulation Products. Applied Optics, 32, 2455-2460. https://doi.org/10.1364/AO.32.002455

[7] Al-Obaidy, H. (2005) Construction of an Acousto-Optic Deflector and Its Application in Bitable Devices. MS.C Thesis, University of Technology, Iraq, Baghdad.

[8] Zadorin, A. and Sharangovich, S. (1986) Strong Acousto-Optic Interaction in the Field of a Focused Acoustic Wave. Radio-Physics and Quantum Electronics, 29, 616-625. https://doi.org/10.1007/BF01034152 